JouRnal OF Universal Mathematics

Vol.1 No.2 PP.155-165 (2018)

ISSN-2618-5660

\title{
CONTROLLED FUZZY EVOLUTION EQUATIONS
}

\author{
S. MELLIANI, A. EL ALLAOUI, AND L. S. CHADLI
}

\begin{abstract}
This paper is concerned with controlled fuzzy nonlinear evolution equations of the form

$$
u^{\prime}(t)=A u(t)+f(t, u(t), u(\rho(t)))+B(t) c(t), \quad t \in\left[t_{0}, t_{1}\right] ; \quad u\left(t_{0}\right)=u_{0}
$$

. Where $c(t) \in E^{1}$ is a control, $A$ generate a fuzzy semigroup and $B$ : $\left[t_{0}, t_{1}\right] \longrightarrow \mathcal{L}\left(E^{1}\right)$. We use the fuzzy strongly continuous semigroups theory to prove the existence, uniqueness and some properties of mild solutions.
\end{abstract}

\section{INTRODUCTION}

The theory of fuzzy sets has lately years been an object of increasing interest because of its vast applicability in several fields include mechanics, electrical engineering, processing signals and in more and more fields. Therefore, it draws a wide attention of the researchers in the recent years.

In this paper, we consider the controlled fuzzy nonlinear evolution equations of the form

$$
\left\{\begin{array}{l}
u^{\prime}(t)=A u(t)+f(t, u(t), u(\rho(t)))+B(t) c(t), \quad t \in I \\
u\left(t_{0}\right)=u_{0}
\end{array}\right.
$$

where $I=\left[t_{0}, t_{1}\right]$ be an interval of the real line and $E^{1}$ be the fuzzy metric space. provided some conditions on the functions $f: I \times E^{1} \times E^{1} \rightarrow E^{1}, \rho: I \longrightarrow I$, $c(t) \in E^{1}$ a control, $B \in \mathcal{C}\left(I, \mathcal{L}\left(E^{1}\right)\right)$ and $A$ is the generator of a strongly continuous fuzzy semigroup. The aim of our paper is to study the existence of mild solution of (1) based on fuzzy strongly continuous semigroups theory (see $[3,10]$ ).

Note that Kaleva [9] discussed the properties of differentiable fuzzy set-valued mappings by means of the concept of $H$-differentiability due to Puri and Ralescu [14], gave the existence and uniqueness theorem for a solution of the fuzzy differential equation

$$
u^{\prime}(t)=f(t, u(t)) ; \quad u(0)=u_{0}
$$

when $f: I \times E^{n} \rightarrow E^{n}$ satisfies the Lipschitz condition.

Date: July 01, 2018, accepted.

2000 Mathematics Subject Classification. 49N05, 34A07, 47D03, 35C15.

Key words and phrases. Control, Fuzzy differential equations, Fuzzy strongly continuous semigroups, Mild solution. 
In [10], Said Melliani, El Hassan Eljaoui and Lalla Saadia Chadli studied, with more details, the existence and uniqueness of mild solution for the fuzzy differential equation

$$
u^{\prime}(t)=A u(t)+f(t, u(h(t))) ; \quad u(0)=u_{0}+(-1) g(u)
$$

In [4] Bhaskar Dubey and Raju K. George studied the linear time-invariant systems with fuzzy initial condition

$$
u^{\prime}(t)=A u(t)+B c(t), \quad u\left(t_{0}\right)=u_{0} .
$$

where $c(t) \in\left(E^{1}\right)^{m}$ a control and $A, B$, are $n \times n, n \times m$ real matrices, respectively, $t_{0} \geq 0$. ather parallel resuls $[5,6]$.

\section{Preliminaries}

Let $\mathcal{P}_{K}\left(\mathbb{R}^{n}\right)$ denote the family of all nonempty compact convex subsets of $\mathbb{R}^{n}$ and define the addition and scalar multiplication in $\mathcal{P}_{K}\left(\mathbb{R}^{n}\right)$ as usual. Let $A$ and $B$ be two nonempty bounded subsets of $\mathbb{R}^{n}$. The distance between $A$ and $B$ is defined by the Hausdorf metric,

$$
d(A, B)=\max \left\{\sup _{a \in A} \inf _{b \in B}\|a-b\|, \sup _{b \in B} \inf _{a \in A}\|a-b\|\right\}
$$

where \|\| denotes the usual Euclidean norm in $\mathbb{R}^{n}$.

Then it is clear that $\left(\mathcal{P}_{K}\left(\mathbb{R}^{n}\right), d\right)$ becomes a complete and separable metric space (see [14]).

Denote

$$
E^{n}=\left\{u: \mathbb{R}^{n} \longrightarrow[0,1] \mid \mathrm{u} \text { satisfies (i)-(iv) below }\right\}
$$

where

(i) $u$ is normal i.e there exists an $x_{0} \in \mathbb{R}^{n}$ such that $u\left(x_{0}\right)=1$,

(ii) $u$ is fuzzy convex,

(iii) $u$ is upper semicontinuous,

(iv) $[u]^{0}=\operatorname{cl}\left\{x \in \mathbb{R}^{n} / u(x)>0\right\}$ is compact.

For $0<\alpha \leq 1$, denote $[u]^{\alpha}=\left\{t \in \mathbb{R}^{n} / u(t) \geq \alpha\right\}$. Then from (i)-(iv), it follows that the $\alpha$-level set $[u]^{\alpha} \in \mathcal{P}_{K}\left(\mathbb{R}^{n}\right)$ for all $0 \leq \alpha \leq 1$.

According to Zadeh's extension principle, we have addition and scalar multiplication in fuzzy number space $E^{n}$ as follows :

$$
[u+v]^{\alpha}=[u]^{\alpha}+[v]^{\alpha}, \quad[k u]^{\alpha}=k[u]^{\alpha}
$$

where $u, v \in E^{n}, k \in \mathbb{R}^{n}$ and $0 \leq \alpha \leq 1$.

Define $D: E^{n} \times E^{n} \rightarrow \mathbb{R}^{+}$by the equation

$$
D(u, v)=\sup _{0 \leq \alpha \leq 1} d\left([u]^{\alpha},[v]^{\alpha}\right)
$$

where $d$ is the Hausdorff metric for non-empty compact sets in $\mathbb{R}^{n}$.

Then it is easy to see that $D$ is a metric in $E^{n}$. Using the results in [14], we know that

(1) $\left(E^{n}, D\right)$ is a complete metric space;

(2) $D(u+w, v+w)=D(u, v)$ for all $u, v, w \in E^{n}$;

(3) $D(k u, k v)=|k| D(u, v)$ for all $u, v \in E^{n}$ and $k \in \mathbb{R}^{n}$.

If we denote $\|u\|_{\mathcal{F}}=D(u, \tilde{0}), u \in E^{n}$, then $\|u\|_{\mathcal{F}}$ has the properties of an usual norm on $E^{n}$ (see [7]), 
(1) $\|u\|_{\mathcal{F}}=0$ iff $u=\tilde{0}$;

(2) $\|\lambda u\|_{\mathcal{F}}=|\lambda|\|u\|_{\mathcal{F}}$ for all $u \in E^{n}, \lambda \in \mathbb{R}$;

(3) $\|u+v\|_{\mathcal{F}} \leq\|u\|_{\mathcal{F}}+\|v\|_{\mathcal{F}}$ for all $u, v \in E^{n}$;

(4) $D(\alpha u, \beta u) \leq|\alpha-\beta| D(u, \tilde{0})$, for all $\alpha, \beta \geq 0$ or $\alpha, \beta \leq 0, u \in E^{n}$.

On $E^{n}$, we can define the substraction $\Theta$, called the $H$-difference (see [8])as follows $u \ominus v$ has sense if there exists $w \in E^{n}$ such that $u=v+w$.

Denote $\mathcal{C}\left(I, E^{n}\right)=\left\{f: I \longrightarrow E^{n} ; f\right.$ is continuous on $\left.I\right\}$, endowed with the metric

$$
H(u, v)=\sup _{t \in I} D(u(t), v(t))
$$

Then $(\mathcal{C}, H)$ is a complete metric space.

Lets $a, b \in \mathbb{R}, f \in \mathcal{C}\left(I, E^{n}\right)$, if we denote $\|f\|=H(f, \tilde{0})$, then $\|f\|$ has the properties of an usual norm on $E^{n}$ (see [7]),

(1) $\|f\|=0$ if $f=\tilde{0}$;

(2) $\|\lambda f\|=|\lambda|\|f\|$ for all $f \in \mathcal{C}\left(I, E^{n}\right), \lambda \in \mathbb{R}$;

(3) $\|f+g\| \leq\|f\|+\|g\|$ for all $f, g \in \mathcal{C}\left(I, E^{n}\right)$;

(4) $H(\alpha f, \beta f) \leq|\alpha-\beta| H(f, \tilde{0})$, for all $\alpha, \beta \geq 0$ or $\alpha, \beta \leq 0, f \in \mathcal{C}\left(I, E^{n}\right)$.

Definition 1.1. A mapping $F: I \rightarrow E^{n}$ is Hukuhara differentiable at $t_{0} \in I$ if there exists a $F^{\prime}(t) \in E^{n}$ such that the following limits

$$
\lim _{h \rightarrow 0^{+}} \frac{F(t+h) \ominus F(t)}{h} \quad \text { and } \quad \lim _{h \rightarrow 0^{+}} \frac{F(t) \ominus F(t-h)}{h}
$$

exist and equal to $F^{\prime}(t)$.

we recall some measurability, integrability properties for fuzzy set-valued mappings (see [9]).

Definition 1.2. A mapping $F: I \rightarrow E^{n}$ is strongly mesurable if for all $\alpha \in[0,1]$ the set-valued function $F_{\alpha}: I \rightarrow \mathcal{P}_{K}\left(\mathbb{R}^{n}\right)$ defined by $F_{\alpha}(t)=[F(t)]^{\alpha}$ is Lebesgue mesurable.

A mapping $F: I \rightarrow E^{n}$ is called integrably bounded if there exists an integrable function $k$ such that $\|x\| \leq k(t)$ for all $x \in F_{0}(t)$.

Definition 1.3. Let $F: I \rightarrow E^{n}$. Then the integral of $F$ over $I$ denoted by $\int_{I} F(t) d t$ or $\int_{I} F(t) d t$, is defined by the equation

$\left[\int_{I} F(t) d t\right]^{\alpha}=\int_{I} F_{\alpha}(t) d t=\left\{\int_{I} f(t) d t / f: I \rightarrow \mathbb{R}^{n}\right.$ is a mesurable selection for $\left.F_{\alpha}\right\}$ for all $\alpha \in] 0,1]$.

Also, a strongly mesurable and integrably bounded mapping $F: I \rightarrow E^{n}$ is said to be integrable over $I$ if

$$
\int_{I} F(t) d t \in E^{n}
$$

Proposition 1. (Aumann [1]). If $F: I \rightarrow E^{n}$ is strongly measurable and integrably bounded, then $F$ is intergrable.

Proposition 2. ([9]) Let $F, G: I \rightarrow E^{n}$ be integrable and $\lambda \in \mathbb{R}$. Then

(i) $\int_{I}(F(t)+G(t)) d t=\int_{I} F(t) d t+\int_{I} G(t) d t$, 
(ii) $\int_{I} \lambda F(t) d t=\lambda \int_{I} F(t) d t$,

(iii) $D(F, G)$ is integrable,

(iv) $D\left(\int_{I} F(t) d t, \int_{I} G(t) d t\right) \leq \int_{I} D(F, G)(t) d t$.

1.1. Operator theory. We give here a definition of linear operator, which is similar to that given by C. G. Gal and S. G. Gal in [7].

Definition 1.4. $A: E^{n} \longrightarrow E^{n}$ is a linear operator if

$$
\left\{\begin{array}{l}
A(x+y)=A x+A y \\
A(\lambda x)=\lambda A(x)
\end{array}\right.
$$

for all $x, y \in E^{n}, \lambda \in \mathbb{R}$.

Remark 1.5. If $A: E^{n} \longrightarrow E^{n}$ is linear and continuous at $\tilde{0} \in E^{n}$, then the latter does not imply the continuity of $A$ at each $x \in E^{n}$, because in general, we cannot write $x_{0}=\left(x_{0} \ominus x\right)+x$.

However, we can prove the following theorems, which is similar to that given in [7].

Theorem 1.6. If $A: E^{n} \longrightarrow E^{n}$ is linear, then it is continuous at $\tilde{0} \in E^{n}$ if and only if there exists $M>0$ such that

$$
\|A(x)\|_{\mathcal{F}} \leq M\|x\|_{\mathcal{F}}, \forall x \in E^{n} .
$$

Now, for $A: E^{n} \longrightarrow E^{n}$ linear and continuous at $\tilde{0}$, let us denote

$$
\mathcal{M}_{A}:=\left\{M>0 ;\|A(x)\|_{\mathcal{F}} \leq M\|x\|_{\mathcal{F}}, \forall x \in E^{n}\right\}
$$

Furthermore, in the both cases, denote $\mid\|A\|_{\mathcal{F}}=\inf _{M} \mathcal{M}_{A}$.

We have the following :

Theorem 1.7. If $A: E^{n} \longrightarrow E^{n}$ is linear and continuous at $\tilde{0}$, then

$$
\|A(x)\|_{\mathcal{F}} \leq|\|A\||_{\mathcal{F}}\|x\|_{\mathcal{F}} .
$$

for all $x \in E^{n}$ and

$$
|\|A\||_{\mathcal{F}}=\sup \left\{\|A(x)\|_{\mathcal{F}} ; x \in E^{n},\|x\|_{\mathcal{F}} \leq 1\right\} .
$$

Corollary 1.8. If $A: E^{n} \longrightarrow E^{n}$ is additive, positive homogeneous and continuous at $\tilde{0}$, then

$$
\|A(x)\|_{\mathcal{F}} \leq \mid\|A\|\left\|_{\mathcal{F}}\right\| x \|_{\mathcal{F}}, \forall x \in E^{n} .
$$

Next, let us denote

$\mathcal{L}_{0}^{+}\left(E^{n}\right)=\left\{A: E^{n} \longrightarrow E^{n} ; A\right.$ is additive, positive homogeneous and continuous at $\left.\tilde{0}\right\}$, $\mathcal{L}^{+}\left(E^{n}\right)=\left\{A \in \mathcal{L}_{0}^{+}\left(E^{n}\right) ; A\right.$ is continuous at each $\left.x \in E^{n}\right\}$, $\mathcal{L}_{0}\left(E^{n}\right)=\left\{A: E^{n} \longrightarrow E^{n} ; A\right.$ is linear and continuous at $\left.\tilde{0}\right\}$,

and

$$
\mathcal{L}\left(E^{n}\right)=\left\{A \in \mathcal{L}_{0}\left(E^{n}\right) ; A \text { is continuous at each } x \in E^{n}\right\},
$$

We consider the metric $\Phi: \mathcal{L}_{0}^{+}\left(E^{n}\right) \times \mathcal{L}_{0}^{+}\left(E^{n}\right) \longrightarrow \mathbb{R}^{+}$by

$$
\Phi(A, B)=\sup \left\{D(A(x), B(x)) ;\|x\|_{\mathcal{F}} \leq 1\right\}, A, B \in \mathcal{L}_{0}^{+}\left(E^{n}\right) .
$$


Then it is easy to see that $\Phi(A, \tilde{O})=|\|A\||_{\mathcal{F}}, A \in \mathcal{L}_{0}^{+}\left(E^{n}\right)$, where $\tilde{O}: E^{n} \longrightarrow E^{n}$ is given by $\tilde{O}(x)=\tilde{0}, \forall x \in E^{n}$.

We can prove the following theorem, which is similar to that given by C. G. Gal and S. G. Gal in [7].

Theorem 1.9. $\quad(1)\left(\mathcal{L}_{0}^{+}\left(E^{n}\right), \Phi\right),\left(\mathcal{L}_{0}\left(E^{n}\right), \Phi\right),\left(\mathcal{L}^{+}\left(E^{n}\right), \Phi\right)$ and $\left(\mathcal{L}\left(E^{n}\right), \Phi\right)$ are complete metric spaces.

(2) $\Phi(A+B, C+D) \leq \Phi(A, C)+\Phi(B, D)$,

(3) $\Phi(k A, k B)=|k| \Phi(A, B)$,

(4) $\Phi(A, B) \leq|\|A\||_{\mathcal{F}}+\mid\|B\|_{\mathcal{F}}$,

(5) $\Phi(A+B, C) \leq \Phi(A, C)+\Phi(B, C)$,

(6) $\Phi(A+B, \tilde{O}) \leq|\|A\||_{\mathcal{F}}+|\|B\||_{\mathcal{F}}$.

for all $A, B, C \in \mathcal{L}_{0}^{+}\left(E^{n}\right)$.

1.2. Fuzzy strongly continuous semigroups. We give here a definition of a fuzzy semigroups, which is similar to that given in $[2,10,13]$.

Definition 1.10. A family $\{T(t), t \geq 0\}$ of operators from $E^{n}$ into itself is a fuzzy strongly continuous semigroup if

(i) $T(0)=i$, the identity mapping on $E^{n}$,

(ii) $T(t+s)=T(t) T(s)$ for all $t, s \geq 0$,

(iii) the function $g:\left[0, \infty\left[\rightarrow E^{n}\right.\right.$, defined by $g(t)=T(t) x$ is continuous at $t=0$ for all $x \in E^{n}$ i.e

$$
\lim _{t \rightarrow 0^{+}} T(t) x=x
$$

(iv) There exist two constants $R>0$ and $\omega$ such that

$$
D(T(t) x, T(t) y) \leq R e^{\omega t} D(x, y), \quad \text { for } t \geq 0, x, y \in E^{n}
$$

In particular if $R=1$ and $\omega=0$, we say that $\{T(t), t \geq 0\}$ is a contraction fuzzy semigroup.

Remark 1.11. The condition (iii) implies that the function $t \longrightarrow T(t) x$ is continuous on $\left[0, \infty\left[\right.\right.$ for all $x \in E^{n}$.

Definition 1.12. Let $\{T(t), t \geq 0\}$ be a fuzzy strongly continuous semigroup on $E^{n}$ and $x \in E^{n}$. If for $h>0$ sufficiently small, the Hukuhara difference $T(h) x \ominus x$ exits, we define

$$
A x=\lim _{h \rightarrow 0^{+}} \frac{T(h) x \ominus x}{h}
$$

whenever this limit exists in the metric space $\left(E^{n}, D\right)$. Then the operator $A$ defined on

$$
D(A)=\left\{x \in E^{n}: \lim _{h \rightarrow 0^{+}} \frac{T(h) x \ominus x}{h} \text { exists }\right\} \subset E^{n}
$$

is called the infinitesimal generator of the fuzzy semigroup $\{T(t), t \geq 0\}$.

Lemma 1.13. Let $A$ be the generator of a fuzzy semigroup $\{T(t), t \geq 0\}$ on $E^{n}$, then for all $x \in E^{n}$ such that $T(t) x \in D(A)$ for all $t \geq 0$, the mapping $t \rightarrow T(t) x$ is differentiable and

$$
\frac{d}{d t}(T(t) x)=A T(t) x, \quad \forall t \geq 0
$$


Example 1.14. We define on $E^{n}$ the family of operator $\{T(t), t \geq 0\}$ by

$$
T(t) x=e^{k t} x, k \in \mathbb{R} .
$$

For $k \geq 0,\{T(t), t \geq 0\}$ is a fuzzy strongly continuous semigroup on $E^{n}$, and the linear operator $A$ defined by $A x=k x$ is the infinitesimal generator of this fuzzy semigroup.

Proposition 3. Let $\{T(t), t \geq 0\}$ be a fuzzy strongly continuous semigroup on $E^{n}$. Then for all $t \geq 0$ we have $T(t) \in \mathcal{L}\left(E^{n}\right)$.

Proof. Lets $x, y \in E^{n}$, the condition (iv) in the definition 2.5 implies that, there exist two constants $M>0$ and $\omega$ such that

$$
D(T(t) x, T(t) y) \leq R e^{\omega t} D(x, y)
$$

Or, $D(x, y) \longrightarrow 0$ as $x \rightarrow y$. Then $D(T(t) x, T(t) y) \longrightarrow 0$ as $x \rightarrow y$.

which implies $T(t) \in \mathcal{L}\left(E^{n}\right)$, for all $t \geq 0$.

\section{Main Results}

To begin our discussion, we need to introduce the concept of mild solution for the problem (1), provided $f:\left[t_{0}, t_{1}\right] \times E^{1} \rightarrow E^{1}, \rho:\left[t_{0}, t_{1}\right] \longrightarrow\left[t_{0}, t_{1}\right], B:\left[t_{0}, t_{1}\right] \longrightarrow$ $\mathcal{L}\left(E^{1}\right), A$ is the generator of a strongly continuous fuzzy semigroup. the input $c(t) \in E^{1}$ for each $t \in\left[t_{0}, t_{1}\right]$ and $c($.$\left.) is fuzzy-integrable (see [9, ?]\right)$ in $\left[t_{0}, t_{1}\right]$. Therefore, it is important to understand the structure of the solutions of (1).

Definition 2.1. We say that $u$ is a mild solution of the equation (1) if

(i) $u \in \mathcal{C}\left(\left[t_{0}, t_{1}\right], E^{1}\right), u(t) \in D(A)$ for all $t \in\left[t_{0}, t_{1}\right]$;

(ii) and $u(t)=T\left(t-t_{0}\right) u_{0}+\int_{t_{0}}^{t} T(t-s)(f(s, u(s), u(\rho(s))+B(s) c(s)) d s$, for all $t \in\left[t_{0}, t_{1}\right]$.

We give here a definition of the Controllability, which is similar to that given by Bhaskar Dubey, Raju K. George in [4].

Definition 2.2. The problem (1) with fuzzy initial condition $u(0)=u_{0} \in E^{1}$ is said to be controllable to a fuzzy-state $u_{1} \in E^{1}$ at $t_{2}>t_{0}$ if there exists a fuzzyintegrable control $c(t) \in E^{1}$ for $t \in\left[t_{0}, t_{1}\right]$ such that the solution of problem (1) with this control satisfies $u\left(t_{2}\right)=u_{1}$.

$$
\text { Let } N=\sup _{t \in\left[t_{0}, t_{1}\right]} R e^{\omega t} \text {. }
$$

We first study the existence and uniqueness of mild solutions using the fixed point argument.

Suppose the assumptions:

$\left(\mathbf{H}_{0}\right) A$ is the infinitesimal generator of a strongly continuous fuzzy semigroup $\{T(t), t \geq 0\}$ on $E^{1}$.

$\left(\mathbf{H}_{1}\right) . f:\left[t_{0}, t_{1}\right] \times E^{1} \rightarrow E^{1}$ is continuous and there exist two constants $L_{1}, L_{2}>0$ such that

$D\left(f(t, x, y), f\left(t, x^{\prime}, y^{\prime}\right)\right) \leq L_{1} D(x, y)+L_{2} D\left(x^{\prime}, y^{\prime}\right), \quad \forall t \in\left[t_{0}, t_{1}\right], \quad x, y, x^{\prime}, y^{\prime} \in E^{1}$.

$\left(\mathbf{H}_{2}\right) . \rho:\left[t_{0}, t_{1}\right] \longrightarrow\left[t_{0}, t_{1}\right]$ is continuous and $\rho(t) \leq t$ for all $t \in\left[t_{0}, t_{1}\right]$.

$\left(\mathbf{H}_{3}\right)$. for $t \in\left[t_{0}, t_{1}\right], c(t) \in E^{1}$ is a control and $c($.$) is fuzzy-integrable.$ 
$\left(\mathbf{H}_{4}\right) . B:\left[t_{0}, t_{1}\right] \longrightarrow \mathcal{L}\left(E^{1}\right)$ is continuous.

$\left(\mathbf{H}_{5}\right)$. There exist a constant $M>0$ such that

$D(B(t) x, B(t) y) \leq M D(x, y)$ for all $x, y \in E^{1}, t \in\left[t_{0}, t_{1}\right]$.

Theorem 2.3. Assume that the conditions $\left(\boldsymbol{H}_{0}\right)-\left(\boldsymbol{H}_{4}\right)$ are satisfied. Then for all $u_{0} \in E^{1}$ such that $T(t) u_{0} \in E^{1}$ for all $t \geq 0$, the problem (1) has a unique mild solution on $\left[t_{0}, t_{1}\right]$.

Proof. Transform the problem (1) into a fixed point problem. Consider the operator $\Gamma: \mathcal{C}\left(\left[t_{0}, t_{1}\right] ; E^{1}\right) \longrightarrow \mathcal{C}\left(\left[t_{0}, t_{1}\right] ; E^{1}\right)$ defined as

$$
\Gamma u(t)=T\left(t-t_{0}\right) u_{0}+\int_{t_{0}}^{t} T(t-s)(f(s, u(s), u(\rho(s)))+B(s) u(s)) .
$$

$\Gamma$ is well defined and maps $\mathcal{C}\left(\left[t_{0}, t_{1}\right] ; E^{1}\right)$ into itself. Indeed

For $u \in E^{1}, t \in\left[t_{0}, t_{1}\right]$ and $h$ very small, we have

$$
\begin{aligned}
D(\Gamma u(t+h), \Gamma u(t))= & D\left(T\left(t+h-t_{0}\right) u_{0}+\int_{t_{0}}^{t+h} T(t+h-s)(f(s, u(s), u(\rho(s)))+B(s) c(s)) d s\right. \\
& \left.T\left(t-t_{0}\right) u_{0}+\int_{t_{0}}^{t} T(t-s)(f(s, u(s), u(\rho(s)))+B(s) c(s)) d s\right) \\
\leq & D\left(T\left(t+h-t_{0}\right) u_{0}, T\left(t-t_{0}\right) u_{0}\right) \\
& +D\left(\int_{t_{0}}^{t+h} T(t+h-s)(f(s, u(s), u(\rho(s)))+B(s) c(s)) d s\right. \\
& \left.\int_{t_{0}}^{t} T(t-s)(f(s, u(s), u(\rho(s)))+B(s) c(s)) d s\right) \\
\leq & N D\left(T(h) u_{0}, u_{0}\right)+\int_{t_{0}}^{t_{0}+h} D(T(t-h+s)(f(s, u(s), u(\rho(s)))+B(s) c(s)) d s, \tilde{0}) d s \\
& +N \int_{t_{0}}^{t} D(f(s+h, u(s+h), u(\rho(s+h)), f(s, u(s), u(\rho(s)))) d s \\
& +N \int_{t_{0}}^{t} D(B(s+h) c(s+h), B(s) c(s)) d s .
\end{aligned}
$$

It is obviously that

$D\left(T(h) u_{0}, u_{0}\right) \rightarrow 0, \quad \int_{t_{0}}^{t_{0}+h} D(T(t-h+s)(f(s, u(s), u(\rho(s)))+B(s) c(s)) d s, \tilde{0}) d s \rightarrow 0$.

And by the dominated convergence theorem we have

$$
\int_{t_{0}}^{t} D(f(s+h, u(s+h), u(\rho(s+h)), f(s, u(s), u(\rho(s)))) d s \rightarrow 0, \quad .
$$

and $\int_{t_{0}}^{t} D(B(s+h) c(s+h), B(s) c(s)) d s \rightarrow 0$

From above, we infer that $\Gamma u \in \mathcal{C}\left(\left[t_{0}, t_{1}\right] ; E^{1}\right)$, for all $u \in \mathcal{C}\left(\left[t_{0}, t_{1}\right] ; E^{1}\right)$. 
For $t \in\left[t_{0}, t_{1}\right], u, v \in E^{1}$, we have

$$
\begin{aligned}
D(\Gamma u(t), \Gamma v(t))= & D\left(T\left(t-t_{0}\right) u_{0}+\int_{t_{0}}^{t} T(t-s)(f(s, u(s), u(\rho(s)))+B(s) c(s)) d s\right. \\
& \left.T\left(t-t_{0}\right) u_{0}+\int_{t_{0}}^{t} T(t-s)(f(s, v(s), v(\rho(s)))+B(s) c(s)) d s\right) \\
\leq & D\left(\int_{t_{0}}^{t} T(t-s)(f(s, u(s), u(\rho(s)))+B(s) c(s)) d s\right. \\
& \left.\int_{t_{0}}^{t} T(t-s)(f(s, v(s), v(\rho(s)))+B(s) c(s)) d s\right) \\
\leq & N \int_{t_{0}}^{t} D(f(s, u(s), u(\rho(s)))+B(s) c(s), f(s, v(s), v(\rho(s)))+B(s) c(s)) d s \\
\leq & N \int_{t_{0}}^{t} D(f(s, u(s), u(\rho(s))), f(s, v(s), v(\rho(s)))) d s \\
\leq & N \int_{t_{0}}^{t}\left(L_{1} D(u(s), v(s))+L_{2} D(u(\rho(s)), v(\rho(s)))\right) d s \\
\leq & \left(L_{1}+L_{2}\right) N\left(t-t_{0}\right) H(u, v) .
\end{aligned}
$$

Therefore, we have

$$
\begin{aligned}
D\left(\Gamma^{2} u(t), \Gamma^{2} v(t)\right) & \leq N \int_{t_{0}}^{t}\left(L_{1} D(\Gamma u(s), \Gamma v(s))+L_{2} D(\Gamma u(\rho(s)), \Gamma v(\rho(s)))\right) d s \\
& \leq N \int_{t_{0}}^{t}\left(L_{1}\left(L_{1}+L_{2}\right) N\left(s-t_{0}\right) H(u, v)+L_{2}\left(L_{1}+L_{2}\right) N\left(\rho(s)-t_{0}\right) H(u, v)\right) d s \\
& \leq\left(L_{1}+L_{2}\right) N\left(t-t_{0}\right) H(u, v) .
\end{aligned}
$$

Since $\rho(s) \leq s$, then

$$
\begin{aligned}
D\left(\Gamma^{2} u(t), \Gamma^{2} v(t)\right) & \leq N \int_{t_{0}}^{t}\left(L_{1} D(\Gamma u(s), \Gamma v(s))+L_{2} D(\Gamma u(\rho(s)), \Gamma v(\rho(s)))\right) d s \\
& \leq N \int_{t_{0}}^{t}\left(L_{1}\left(L_{1}+L_{2}\right) N\left(s-t_{0}\right) H(u, v)+L_{2}\left(L_{1}+L_{2}\right) N\left(s-t_{0}\right) H(u, v)\right) d s \\
& =\left(L_{1}+L_{2}\right)^{2} N^{2} H(u, v) \int_{t_{0}}^{t}\left(s-t_{0}\right) d s \\
& =\frac{\left(L_{1}+L_{2}\right)^{2} N^{2}\left(t-t_{0}\right)^{2}}{2} H(u, v) .
\end{aligned}
$$

By inference, we have for all $m>0$

$$
D\left(\Gamma^{m} u(t), \Gamma^{m} v(t)\right) \leq \frac{\left(\left(L_{1}+L_{2}\right) N\left(t-t_{0}\right)\right)^{m}}{m !} H(u, v), \forall t \in\left[t_{0}, t_{1}\right] .
$$

Which means that

$$
H\left(\Gamma^{m} u, \Gamma^{m} v\right) \leq \frac{\left(\left(L_{1}+L_{2}\right) N\left(t-t_{0}\right)\right)^{m}}{m !} H(u, v), \forall t \in\left[t_{0}, t_{1}\right] .
$$

Since

$$
\lim _{m \rightarrow+\infty} \frac{\left(\left(L_{1}+L_{2}\right) N\left(t-t_{0}\right)\right)^{m}}{m !}=0 .
$$

Then there exists $p>0$ such that

$$
\frac{\left(\left(L_{1}+L_{2}\right) N\left(t-t_{0}\right)\right)^{p}}{p !}<1 .
$$


which shows that $\Gamma^{p}$ is a contraction. So there exists a unique fixed point $u \in \mathcal{C}\left(\left[t_{0}, t_{1}\right] ; E^{1}\right)$ such that $\Gamma^{p} u=u$.

Which implies that $\Gamma^{p}(\Gamma u)=\Gamma\left(\Gamma^{p} u\right)=\Gamma u$. Since $u$ is unique, then $\Gamma u=u$. It follows that $u$ is the unique mild solution of the problem (1).

The next theorem provides a correspondence between the continuity of solution and the continuity of control.

Theorem 2.4. Let $\left\{c_{n}, n \geq 0\right\} \subset E^{1}$ be a sequence of controls as in $\left(\boldsymbol{H}_{3}\right)$ such that $\lim _{n \rightarrow+\infty} c_{n}=c$. Suppose that the conditions $\left(\boldsymbol{H}_{0}\right)-\left(\boldsymbol{H}_{5}\right)$ hold. Let $u^{c_{n}}$ be the mild solution for (1) corresponding to $c_{n}$ and $u^{c}$ be the mild solution corresponding to $c$. If $N\left(L_{1}+L_{2}\right)\left(t_{1}-t_{0}\right)<1$, then $\lim _{n \rightarrow+\infty} u^{c_{n}}=u^{c}$.

Proof. For $t \in\left[t_{0}, t_{1}\right]$ we have

$$
\begin{aligned}
D\left(u^{c_{n}}(t), u^{c}(t)\right)= & D\left(T\left(t-t_{0}\right) u_{0}+\int_{t_{0}}^{t} T(t-s)\left(f\left(s, u^{c_{n}}(s), u^{c_{n}}(\rho(s))\right)+B(s) c_{n}(s)\right) d s\right. \\
& \left.T\left(t-t_{0}\right) u_{0}+\int_{t_{0}}^{t} T(t-s)\left(f\left(s, u^{c}(s), u^{c}(\rho(s))\right)+B(s) c(s)\right) d s\right) \\
\leq & D\left(\int_{t_{0}}^{t} T(t-s)\left(f\left(s, u^{c_{n}}(s), u^{c_{n}}(\rho(s))\right)+B(s) c_{n}(s)\right) d s\right. \\
& \left.\int_{t_{0}}^{t} T(t-s)\left(f\left(s, u^{c}(s), u^{c}(\rho(s))\right)+B(s) c(s)\right) d s\right) \\
\leq & N \int_{t_{0}}^{t} D\left(f\left(s, u^{c_{n}}(s), u^{c_{n}}(\rho(s))\right)+B(s) c_{n}(s), f\left(s, u^{c}(s), u^{c}(\rho(s))\right)+B(s) c(s)\right) d s \\
\leq & N \int_{t_{0}}^{t}\left(L_{1} D\left(u^{c_{n}}(s), u^{c}(s)\right)+L_{2} D\left(u^{c_{n}}(\rho(s)), u^{c}(\rho(s))\right)\right) d s \\
& +N \int_{t_{0}}^{t} D\left(B(s) c_{n}(s), B(s) c(s)\right) d s \\
\leq & N\left(L_{1}+L_{2}\right)\left(t_{1}-t_{0}\right) H\left(u^{c_{n}}, u^{c}\right)+M N\left(t_{1}-t_{0}\right) H\left(c_{n}, c\right) .
\end{aligned}
$$

Which implies that

$$
H\left(u^{c_{n}}, u^{c}\right) \leq \frac{M N\left(t_{1}-t_{0}\right)}{1-N\left(L_{1}+L_{2}\right)\left(t_{1}-t_{0}\right)} H\left(c_{n}, c\right)
$$

Since $c_{n} \rightarrow c$, hence $u^{c_{n}} \rightarrow u^{c}$ as $n \rightarrow+\infty$.

we establish the following result about continuous dependence of a mild Solution.

Theorem 2.5. Suppose that the conditions $\left(\boldsymbol{H}_{0}\right)-\left(\boldsymbol{H}_{4}\right)$ hold. Lets $u$ and $v$ be mild solutions of (1) on $\left[t_{0}, t_{1}\right]$ corresponding to $u_{0}$ and $v_{0}$ respectively. If $L_{2} N\left(t_{1}-t_{0}\right) e^{L_{1} N\left(t_{1}-t_{0}\right)}<1$, then

$$
H(u, v) \leq \frac{N}{e^{L_{1} N\left(t_{0}-t_{1}\right)}+L_{2} N\left(t_{0}-t_{1}\right)} D\left(u_{0}, v_{0}\right)
$$


Proof. for $t \in\left[t_{0}, t_{1}\right]$, we have

$$
\begin{aligned}
D(u(t), v(t))= & D\left(T\left(t-t_{0}\right) u_{0}+\int_{t_{0}}^{t} T(t-s)(f(s, u(s), u(\rho(s)))+B(s) c(s)) d s,\right. \\
& \left.T\left(t-t_{0}\right) v_{0}+\int_{t_{0}}^{t} T(t-s)(f(s, v(s), v(\rho(s)))+B(s) c(s))\right) d s \\
\leq & N D\left(u_{0}, v_{0}\right)+N \int_{t_{0}}^{t} D(f(s, u(s), u(\rho(s))), f(s, v(s), v(\rho(s)))) d s \\
\leq & N D\left(u_{0}, v_{0}\right)+N \int_{t_{0}}^{t}\left(L_{1} D(u(s), v(s))+L_{2} D(u(\rho(s)), v(\rho(s)))\right) d s \\
\leq & N D\left(u_{0}, v_{0}\right)+L_{2} N\left(t_{1}-t_{0}\right) H(u, v)+L_{1} N \int_{t_{0}}^{t} D(u(s), v(s)) d s
\end{aligned}
$$

By using Gronwall inequality, we find that

$$
\begin{aligned}
D(u(t), v(t)) & \leq\left(N D\left(u_{0}, v_{0}\right)+L_{2} N\left(t_{1}-t_{0}\right) H(u, v)\right) e^{L_{1} N\left(t-t_{0}\right)} \\
& \leq\left(N D\left(u_{0}, v_{0}\right)+L_{2} N\left(t_{1}-t_{0}\right) H(u, v)\right) e^{L_{1} N\left(t_{1}-t_{0}\right)}, \quad \forall t \in\left[t_{0}, t_{1}\right] .
\end{aligned}
$$

which implies that

$$
H(u, v) \leq\left(N D\left(u_{0}, v_{0}\right)+L_{2} N\left(t_{1}-t_{0}\right) H(u, v)\right) e^{L_{1} N\left(t_{1}-t_{0}\right)} .
$$

Finally, we get

$$
H(u, v) \leq \frac{N}{e^{L_{1} N\left(t_{0}-t_{1}\right)}+L_{2} N\left(t_{0}-t_{1}\right)} D\left(u_{0}, v_{0}\right) .
$$

\section{Conclusion}

In order to describe a random evolution of the temperature of the rode using a control, we consider a controlled fuzzy evolution equation. By using operator semigroup of fuzzy sets theory, we obtain existence results. In addition, future work includes expanding the idea signalized in this work and introducing obsevability and generalize other works $[11,12]$. This is a fertile field with vast research projects, which can lead to numerous theories and applications. We plan to devote significant attention to this direction. And we intend to investigate the applications which are based on experimental data (real world problems) of the proposed theory.

\section{REFERENCES}

[1] R. J. Aumann, Integrals of set-valued functions, J. Math. Anal. Appl., (1965), 1-12.

[2] A. El Allaoui, S. Melliani and L. S. Chadli, Fuzzy dynamical systems and Invariant attractor sets for fuzzy strongly continuous semigroups, Journal of Fuzzy Set Valued Analysis 2016 No.2 (2016) 148-155.

[3] A. El Allaoui, S. Melliani, L. S. Chadli, Fuzzy $\alpha$-semigroups of operators, General Letters in Mathematics Vol 2 (2) (2017)42-49.

[4] Bhaskar Dubey, Raju K. George, Controllability of Linear Time-invariant Dynamical Systems with Fuzzy Initial Condition, Proceedings of the World Congress on Engineering and Computer Science (2013), 23-25.

[5] Bhaskar Dubey and Raju K. George, Estimation of controllable initial fuzzy states of linear time-invariant dynamical systems, Communications in Computer and Information Science, Springer, (2012), 316-324.

[6] Y. Feng, L. Hua, On the quasi-controllability of continuous-time dynamic fuzzy control systems, Chaos, Solitons \& Fractals (2006), 177-188. 
[7] C. G. Gal and S. G. Gal, Semigroups of Operators on Spaces of Fuzzy-Number-Valued Functions with Applications to Fuzzy Differential Equations, arXiv:1306.3928v1 (2013).

[8] M. Hukuhara, Integration des applications measurables dont la valeur est un compact convexe, Funk. Ekvacioj (1967), 207-223.

[9] O. Kaleva, Fuzzy Differentiel Equations, Fuzzy Sets and Systems. (1987), 24: 301-317.

[10] Said Melliani, El Hassan Eljaoui and Lalla Saadia Chadli, Fuzzy Differential Equation With Nonlocal Conditions And Fuzzy semigroups, Advances in Difference Equations (2016).

[11] S. Melliani, L. S. Chadli, A. El Allaoui, Periodic boundary value problems for controlled nonlinear impulsive evolution equations on Banach spaces. International Journal of Nonlinear Analy-sis and Applications, 8(1) (2017), 301-314.

[12] S. Melliani, A. El Allaoui and L. S. Chadli, A general classof periodic boundary value problems for controlled nonlinear impulsive evolution equations on Banach spaces, Advances in Difference Equations (2016): 290.

[13] S. Melliani, A. El Allaoui and L. S. Chadli , Relation Between Fuzzy Semigroups and Fuzzy Dynamical Systems, Nonlinear Dynamics and Systems Theory, 17 (1) (2017) 60-69.

[14] M. L. Puri, D. A. Ralescu. Fuzzy random variables, J. Math. Anal. Appl. (1986), 114: 409422.

(author one) Laboratoire de Mathématiques Appliquées \& Calcul Scientifique, Sultan Moulay Slimane University, BP 523, 23000, Beni Mellal, Morocco

E-mail address, author one: said.melliani@gmail.com

(author two) Laboratoire de Mathématiques Appliquées \& Calcul Scientifique, Sultan Moulay Slimane University, BP 523, 23000, Beni Mellal, Morocco

E-mail address, author two: elallaoui199@gmail.com

(author three) Laboratoire de Mathématiques Appliquées \& Calcul Scientifique, Sultan Moulay Slimane University, BP 523, 23000, Beni Mellal, Morocco

E-mail address, author three: sa.chadli@yahoo.fr 